\title{
Utilização de Blockchain na Rastreabilidade da Cadeia Produtiva do Leite
}

\author{
Ronan D. Mendonça ${ }^{1}$, Otávio S. Gomes ${ }^{1}$, Pollyanna C. Pereira ${ }^{1}$ \\ Alex Borges Vieira ${ }^{2}$, José Augusto Nacif ${ }^{1}$ \\ ${ }^{1}$ Instituto de Ciências Exatas e Tecnológicas, Campus UFV-Florestal \\ Universidade Federal de Viçosa \\ ${ }^{2}$ Departamento de Ciência da Computação, Universidade Federal de Juiz de Fora \\ \{ronan.dutra, otavio.s.gomes, pollyanna.cardoso, jnacif\}eufv.br \\ alex.borges@ufje.edu.br
}

\begin{abstract}
Resumo. A tecnologia blockchain está modificando a forma de gerenciar cadeias de suprimentos e tem potencial para gerar efeitos benéficos no ramo alimentício aumentando a confiabilidade dos dados de rastreabilidade. Este trabalho apresenta uma arquitetura para rastrear a cadeia produtiva do leite que utiliza a tecnologia blockchain para armazenar e validar os dados coletados nos vários pontos de controle do produto, oferecendo maior confiabilidade $e$ transparência às transações. A solução é viável no cenário de rastreabilidade do leite, uma vez que a integração dos componentes foi realizada de forma adequada às necessidades e apresenta baixo impacto nos serviços já existentes.
\end{abstract}

\section{Introdução}

A rastreabilidade da cadeia de suprimentos tem como premissa a integridade e a transparência dos dados que estão sendo salvos e compartilhados [Cui 2020]. Além disso, a rastreabilidade é uma forma de prevenir a entrada de alimentos sem qualidade ou segurança, reduzir o volume de devolução de produtos (recall), estabelecer responsabilidades e simplificar a localização de problemas como identificação dos locais/etapas que estão fora da normalidade [Chapaval and Alves 2008].

Nesse contexto, a aplicação da tecnologia da blockchain é promissora para rastreabilidade da cadeia de suprimentos das diversas cadeias agroindustriais. Na cadeia do leite e derivados, a blockchain é uma tecnologia que possibilita resolver os principais problemas devido às capacidades de integração e automação, conectando todos os agentes e pontos da cadeia, automatizando ações. A tecnologia possibilita a rastreabilidade de toda a cadeia produtiva do leite e possibilita também incluir, de maneira imediata e dinâmica, órgãos reguladores para certificarem esse processo dentro da plataforma [Shingh et al. 2020].

Esse trabalho tem como objetivo oferecer uma solução tecnológica relacionada ao rastreamento da cadeia de suprimento da indústria de laticínios. Apresentamos uma arquitetura distribuída, baseada em blockchain, para conceber a construção de uma aplicação de rastreabilidade da produção leiteira. O rastreamento proposto abrange a utilização de dados capturados por sensores e interligados por meio da blockchain, que servirão para o acompanhamento de cada passo dado pelo suprimento até o seu beneficiamento. As 
informações acerca de cada suprimento serão armazenadas de forma imutável e segura na blockchain. Com isto, o consumidor poderá conhecer a origem, qualidade e o percurso por onde o suprimento transitou, até a disponibilização do produto final.

O restante deste trabalho está organizado da seguinte forma. A seção 2 apresenta os trabalhos relacionados. Na seção 3 nós apresentamos uma arquitetura de utilização de blockchain para a rastreabilidade de produtos e apresentamos um estudo experimental da rastreabilidade do leite utilizando a blockchain. Na seção 4 discutimos e apresentamos os resultados obtidos. Finalmente, na seção 5 apresentamos as considerações finais e os trabalhos futuros.

\section{Trabalhos relacionados}

A blockchain está sendo utilizada na cadeia de suprimentos para armazenar e compartilhar dados que podem ser verificados na confirmação de sua autenticidade. Segundo [Kshetri 2018], a blockchain pode fornecer transparência através da imutabilidade das transações, o que aumenta a confiança das partes interessadas e gera a rastreabilidade. Os trabalhos apresentados a seguir utilizaram um estudo de caso para a implementação da tecnologia blockchain no contexto de rastreabilidade da cadeia de suprimentos.

Em [Bumblauskas et al. 2020], os autores apresentam uma implementação para rastreamento da produção e entrega de ovos por uma empresa dos EUA. O objetivo do trabalho é verificar a precisão e transparência em aplicar a blockchain na rastreabilidade da cadeia de suprimentos para suprir o consumidor de informações acerca do alimento consumido. Os métodos da implementação utilizaram sensores IoT e a integração da blockchain Hyperledger e uma aplicação web com o intuito de fornecerem uma estrutura de captura de dados e análises relacionados ao impacto de sua implementação.

[Xu et al. 2019] construíram uma aplicação, intitulada originChain, para prover rastreabilidade baseada em blockchain. Os autores utilizaram uma blockchain baseada em Ethereum de forma pública e privada. Eles avaliaram a aplicação de forma quantitativa por meio da latência de leitura e escrita na blockchain, em um banco de dados local e um banco de dados remoto. A discussão em relação aos resultados apontam o desempenho e a privacidade como limitações a serem consideradas e que as blockchain privadas possuem melhores desempenhos em relação às públicas.

O trabalho de [Figorilli et al. 2018], por sua vez, implementa uma arquitetura em blockchain para a rastreabilidade da cadeia de suprimentos de madeira. O sistema de rastreamento foi simulado para obter dados da cadeia de suprimentos que utiliza sensores RFID. Foi utilizado o Workbench Azure Blockchain como infraestrutura para a criação da aplicação blockchain. Segundo os autores, o uso da tecnologia blockchain para rastreabilidade da madeira é viável economicamente e garante confiabilidade, transparência e segurança a produtos sujeitos a fraude.

Neste trabalho, nós propomos uma arquitetura baseada em blockchain para oferecer a interligação e transmissão de dados entre sistemas com tipos de comunicação variados conforme ocorre na cadeia produtiva do leite. Criamos a arquitetura modular e utilizamos a blockchain como elo de ligação entre os diversos módulos do sistema, o que torna simples a adição de novos componentes sem impacto no serviço. 


\section{Arquitetura proposta}

Baseados nos processos da cadeia de suprimentos do leite, definimos um modelo de arquitetura para atender a rastreabilidade em relação ao funcionamento da captação, armazenamento e transporte da produção leiteira. A arquitetura visa interligar os diversos componentes em um sistema de gerenciamento da cadeia de suprimentos para garantir que os usuários tenham confiabilidade nos dados coletados durante o processo de produção. Um exemplo de implementação da arquitetura é apresentado nesta seção a fim de validar a aplicabilidade da arquitetura proposta.

O arcabouço foi estruturado para prover uma maior adaptabilidade ao cenário real, fazendo o uso da blockchain para o armazenamento e validação de dados coletados por sensores. A arquitetura é composta por três módulos principais, sendo que o Módulo rastreabilidade e o Módulo cliente comunicam diretamente com a blockchain. Já o Módulo sensores provê suporte à comunicação dos sensores externos ao sistema. Diferentes tipos de sensores podem ser usados para realizar esse monitoramento em tempo real, com a atualização das informações em rede. Essas informações podem incluir a localização da unidade, a quantidade de unidades em cada lote, parâmetros da qualidade, além de qualquer informação relevante para fins comerciais e gerenciais [Shingh et al. 2020]. A Figura 1 apresenta a cadeia produtiva do leite com relação ao sensoriamento e ao armazenamento dos dados coletados para análise e rastreabilidade em todos os pontos de controle.

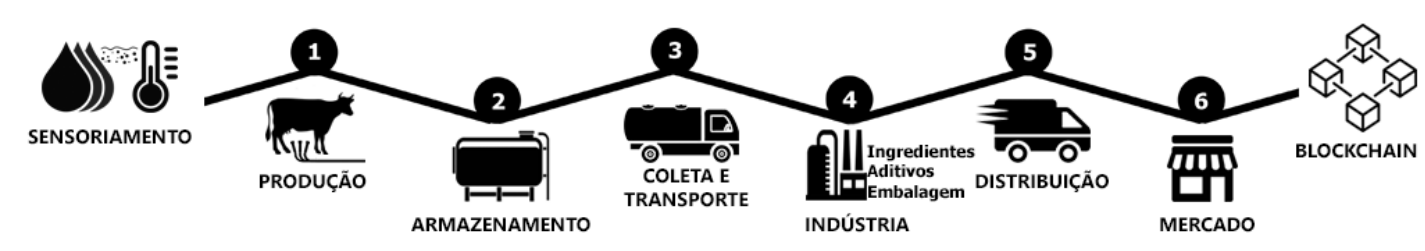

Figura 1. Cadeia produtiva do leite - Adaptada de [Shingh et al. 2020]

A blockchain foi utilizada como um banco de dados distribuído e também para a validação de acessos. Os contratos inteligentes são programas inseridos na blockchain que possibilitam a execução e manipulação de transações com condições programáveis. Eles foram utilizados para organizar o processo de manipulação dos dados.

O Módulo Rastreabilidade é responsável por toda interoperabilidade entre a captação dos dados coletados no decorrer da cadeia de suprimentos e a sua disponibilização na blockchain. Para isso, o módulo primeiramente registra os membros do módulo sensores aptos a utilizarem o sistema. Após o registro, o módulo é capaz de intermediar a gravação dos dados capturados dos produtos por cada membro e a blockchain.

O Módulo Cliente fica encarregado em prover acesso público aos dados registrados pelo módulo rastreabilidade. Por meio dele, o consumidor final ou qualquer outro interessado na rastreabilidade do produto, poderá solicitar a visualização dos dados coletados. O histórico da cadeia de um determinado produto poderá ser acessado a qualquer momento por este módulo, não necessitando assim, o produto passar por todos os pontos de coletas de dados. 


\subsection{Aplicabilidade da arquitetura}

\subsubsection{Implementação dos componentes da arquitetura}

A implementação da arquitetura tem seus pilares baseados em diferentes estruturas tecnológicas. Foram utilizadas uma rede privada baseada na rede blockchain Ethereum, funcionando como banco de dados seguro e confiável de informações, contratos inteligentes, para o gerenciamento das informações salvas na rede blockchain, uma aplicação para geração e inserção de dados na rede e uma aplicação desenvolvida para o rastreio do produto.

O componente Blockchain foi utilizado como base para armazenamento dos dados relacionados ao produto de forma privada. A forma pública da rede blockchain foi utilizada para armazenar o hash das informações dos produtos e os membros autorizados a inserir os dados dos produtos na rede. Foram desenvolvidos dois contratos inteligentes para executar todas as tarefas necessárias para a rastreabilidade de produtos e realizar a organização de informações. Os contratos foram escritos na linguagem Solidity, compilados e migrados para uma rede pública. O contrato nomeado Inicialização é sequenciado na Figura 2. Ele é responsável por inserir e gerenciar os usuários da rede. Esses usuários são contas na rede privada que podem ser tanto atribuídas a sensores automatizados quanto a pessoas autorizadas a inserir dados dos produtos. Portanto, o contrato Inicialização é utilizado para armazenar esses usuários e posteriormente retornar a validade dos mesmos.

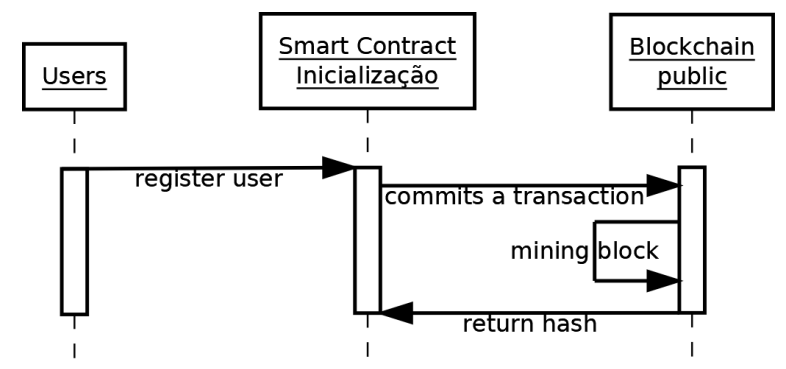

Figura 2. Diagrama Smart Contract Inicialização

O contrato Inserção, por sua vez, é responsável por gerenciar a inserção de informações na rede, assim como verificar a autenticidade de usuários para que esses dados sejam inseridos apenas por endereços válidos. Além disso, é esse contrato que retorna os dados para o rastreio de produtos. O diagrama da Figura 3 mostra a sequência sugerida das trocas de mensagens entre os componentes da aplicação.

O Módulo Rastreabilidade é responsável por permitir a inserção de dados na blockchain. Portanto, foi utilizado a linguagem de programação Python para implementálo e, a partir da biblioteca web3py, foi possível interagir com os contratos inteligentes na rede e inserir e rastrear produtos da cadeia de suprimentos. No Módulo Cliente, que é o módulo responsável por permitir o rastreio de produtos, utilizamos a plataforma de desenvolvimento Android Studio. A aplicação ficou estruturada de forma que na tela inicial é solicitado a inserção do código do produto e um botão rastrear.Ao digitar um código válido e solicitar o rastreio, o aplicativo abre uma nova janela com todas as informações disponíveis na blockchain sobre o produto. 


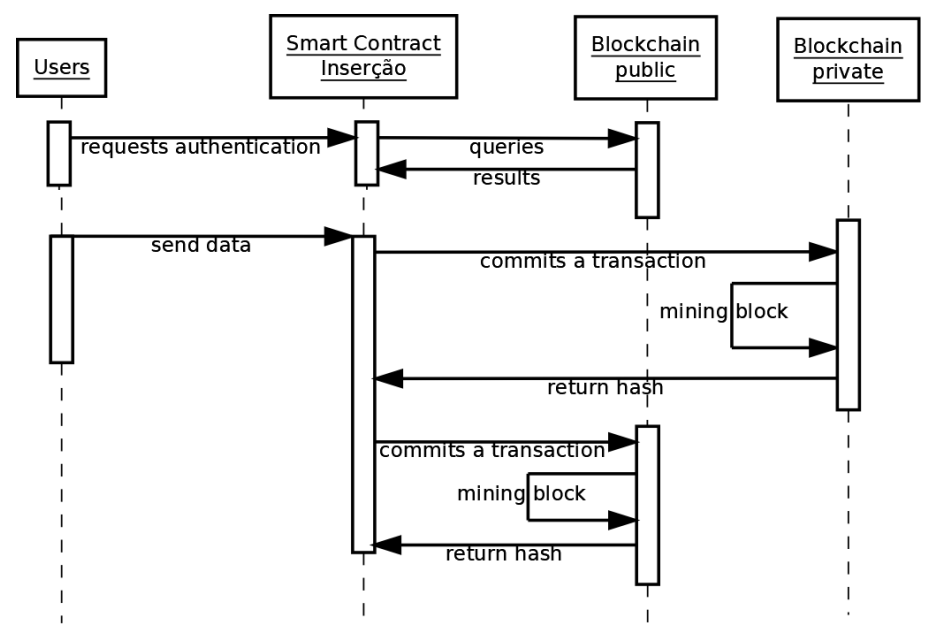

Figura 3. Diagrama Smart Contract Inserção

\section{Avaliação e Resultados}

Para realizar a avalização foram criados dois cenários de execução da solução. O Cenário 1 contendo apenas um nó Ethereum e o Cenário 2 contendo dois nós Ethereum. Os dados capturados durante a execução do experimento foram o Tempo da transação, que é o tempo gasto para efetivar uma transação, e o Tamanho da fila, que é o tamanho da fila de espera a cada inserção de transação.

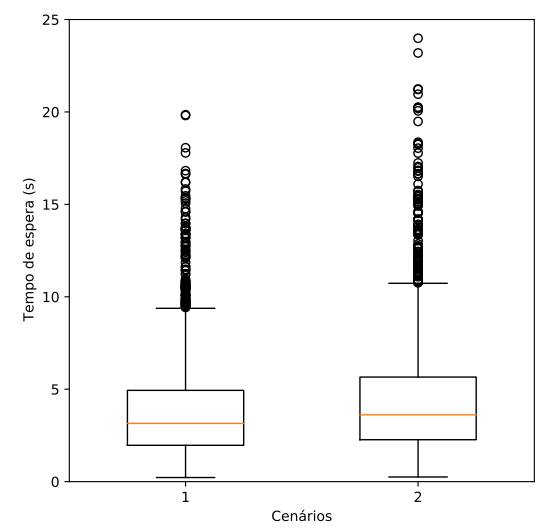

(a) Tempo da transação

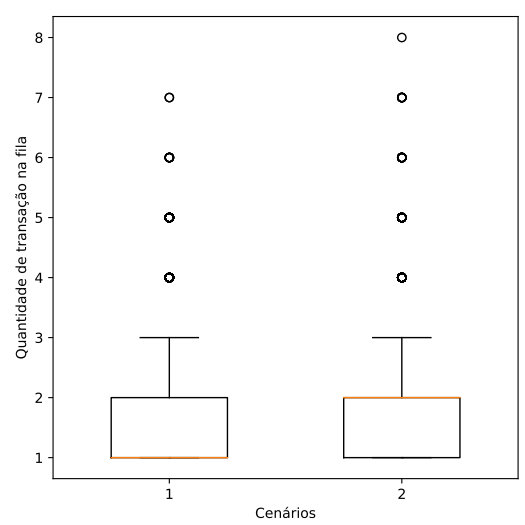

(b) Tamanho da fila

Figura 4. Resultados obtidos nos cenários avaliados

Os resultados apresentados nos gráficos da Figura 4(a) representam o tempo de espera que uma transação levou para ser efetivamente gravada na blockchain. Os valores de média do tempo de espera para o cenário 1 é 3.89 segundos e a mediana é de 3.15 segundos. Para o cenário 2, a média é de 3.61 segundos e a mediana de 4.49 segundos. Como podemos observar, os dois cenários apresentam níveis médios baixos em relação ao tempo de resposta. Esses resultados nos levam a concluir que o tempo de resposta apresentado é compatível com a necessidade de uma aplicação com finalidade de rastrear produtos uma vez que não há necessidades específicas nos requisitos da aplicação para a disponibilização dos dados em tempo real. 
Já nos gráficos da Figura 4(b), são apresentados, para os dois cenários, o tamanho da fila de espera, que é a fila que as transações enfrentam até que seja iniciado o seu processo de mineração. Os valores de média do tamanho da fila de espera para o cenário 1 é 1.74 e a mediana é de 1 . Para o cenário 2, a média é de 1.86 e a mediana de 2 . Os cenários apresentam filas de esperas pequenas, o que não afeta o desempenho em relação ao tempo da efetivação da transação. Porém, em relação à escalabilidade é necessário uma avaliação mais ampla para uma conclusão definitiva.

\section{Considerações finais}

Apresentamos um experimento para validar o uso da blockchain para a rastreabilidade da cadeia produtiva do leite. A solução utilizou a tecnologia blockchain Ethereum para armazenar os dados coletadas dos produtos e hashes criptográficos para validação desses dados. A arquitetura proposta e os componentes utilizados são apresentados de forma a favorecer um acesso facilitado à tecnologia blockchain. Os contratos inteligentes são as soluções bases em blockchain para esse tipo de aplicação, e devem receber uma atenção especial com o intuito de progredir e validar as possibilidade de uso da blockchain em soluções de rastreabilidade da cadeia de suprimentos. Nossas implementações demonstram que o uso da blockchain para a rastreabilidade é viável e pode oferecer transparência nas transações da cadeia de suprimentos do leite. Os próximos passos para esse trabalho envolvem a ampliação da avaliação da metodologia de forma escalável, fazendo com que a disputa pelos recursos sejam avaliadas em grandes demandas.

\section{Agradecimentos}

Os autores agradecem o apoio da CAPES, CNPq e FAPEMIG.

\section{Referências}

Bumblauskas, D., Mann, A., Dugan, B., and Rittmer, J. (2020). A blockchain use case in food distribution: Do you know where your food has been? International Journal of Information Management, 52:102008.

Chapaval, L. and Alves, F. S. F. (2008). Rastreabilidade na produção de leite de cabra: diferencial para um agronegócio sustentável. EMBRAPA Caprinos.

Cui, K. (2020). Optimization of animal product supply chain based on blockchain traceability. Revista Cientifica-Facultad de Ciencias Veterinarias, 30(1).

Figorilli, S., Antonucci, F., Costa, C., Pallottino, F., Raso, L., Castiglione, M., Pinci, E., Del Vecchio, D., Colle, G., Proto, A. R., et al. (2018). A blockchain implementation prototype for the electronic open source traceability of wood along the whole supply chain. Sensors, 18(9):3133.

Kshetri, N. (2018). 1 blockchain's roles in meeting key supply chain management objectives. International Journal of Information Management, 39:80-89.

Shingh, S., Kamalvanshi, V., Ghimire, S., and Basyal, S. (2020). Dairy supply chain system based on blockchain technology. Asian Journal of Economics, Business and Accounting, pages 13-19.

Xu, X., Lu, Q., Liu, Y., Zhu, L., Yao, H., and Vasilakos, A. V. (2019). Designing blockchain-based applications a case study for imported product traceability. Future Generation Computer Systems, 92:399-406. 